\title{
Towards a New Wave of Telerehabilitation Applications
}

\author{
Gilberto Marzano ${ }^{*}$, Luis Ochoa-Siguencia ${ }^{2}$ and Anna Pellegrino ${ }^{3}$ \\ ${ }^{1}$ Rezekne Academy of Technologies, Latvia \\ ${ }^{2}$ The Jerzy Kukuczka Academy of Physical Education in Katowice, Poland \\ ${ }^{3}$ University of Udine, Italy
}

\section{Perspective Article \\ Volume 1 Issue 1}

Received Date: March 14, 2017

Published Date: April 17, 2017

DOI: $10.23880 /$ phoa-16000105

*Corresponding author: Gilberto Marzano, Rezekne Academy of Technologies, Latvia, E-mail: gilberto.marzano@ru.lv

\section{Abstract}

In recent years, new scenarios for experimenting telerehabilitation services have been opening thanks to the diffusion of the new technologies. The revolution brought about by the Internet of Things and Big Data Analytics is having an effect also in the field of telerehabilitation services. The literature has broadened in scope and grown in volume and, in certain aspects, the focus of research has changed in the last few years.

This article examines the major changes that have come about in the field of telerehabilitation, which can essentially be divided into two main strands: low-cost end-user applications, and the integration of telerehabilitation services. We will briefly review the emerging investigations and experimentations in the field of telerehabilitation, analyzing the market trends in the sector and the commercial strategies of companies working in it, and aim to outline the most relevant challenges that exist for the delivery of effective and sustainable telerehabilitation services.

Our opinion is that telerehabilitation currently represents a very promising field, although many questions still remain open, for which concrete and reliable answers are required. In this respect, we focus on a fundamental issue that underlies the field of telerehabilitation services, namely the influence that environment has on the effectiveness of treatment. In short, how can the type of environment affect the results of treatment?

Keywords: Telerehabilitation; Telerehabilitation services; Robot-assisted treatments; Social telerehabilitation

\section{The Telerehabilitation Scenario}

Many different terms are used to designate the application of ICTs in the field of healthcare. The term medical informatics, first coined around 1970, was superseded at the end of the 1990s by eHealth, while, nowadays, telemedicine, tele health, and tele care are all used fairly interchangeably.

The main advantages of Telemedicine in healthcare are evident [1-3]. It is a form of secondary prevention encompassing services dedicated to persons classified as at risk or suffering from chronic diseases (e.g. diabetes or cardiovascular disease) who require a constant monitoring of vital parameters in order to reduce the risk of complications, such as that of blood glucose levels for diabetic patients. Meanwhile, Tele-diagnosis focuses on moving diagnostic information rather than the patient. Although a complete diagnosis cannot be performed exclusively through the use of ICT tools, computer-based systems can effectively support diagnostic processes, for example by giving the possibility of exchanging data amongst specialists and facilitating its communication.

Home health monitoring services utilise ITC-based technology to monitor patients in their homes by means of devices that measure vital data, such as blood pressure, 
glucose levels, pulse, blood oxygen levels, etc., and enable the transmission of this data to clinicians $[4,5]$.

Recently, the concept of telerehabilitation has been introduced to refer to the provision of rehabilitation care at a distance. Telerehabilitation, or e-rehabilitation, is considered a subcomponent of the broader area of telemedicine [6], and can be divided into three main categories: image based telerehabilitation, sensor based telerehabilitation, and telerehabilitation based on virtual technologies [7]. Lately, the notion of social telerehabilitation has been introduced to distinguish the application of ICT to the social rehabilitation sphere $[8,9]$.

Telerehabilitation is widely considered to be advantageous in the treatment of patients. Telerehabilitation services are seen as being a costeffective alternative to traditional rehabilitation services since they can be delivered at a distance, thus reducing the travel costs and difficulties for patients to receive care at a healthcare facility.

The increasing interest in telerehabilitation is closely related to the diffusion of the internet. Indeed, thanks to the internet, all traditional sectors, including healthcare, are going through processes of transformation in order to become more effective and accurate, as well as cheaper and more powerful.

Telerehabilitation solutions have been experimented in many areas, particularly that of rehabilitation following traumatic injury (for assessment, physical therapy, and monitoring).

\section{New Research Perspective}

Research has underlined the potential for social media, mobile phones, and the internet in general, to improve healthcare services by facilitating patient-doctor communication and access to medical reports.

Recently, Artificial Intelligence solutions and Big Data applications are introducing new opportunities to overcome the limitations of the traditional computerbased approach.

Thanks to the developments in technology over the last fifteen years, robots are beginning to be employed for healthcare applications. The use of robots in healthcare is aimed at monitoring patients or assisting them to perform difficult tasks, as well as helping to support them on an emotional and psychosocial level. There are two different classes of healthcare robots: rehabilitation robots and social robots.

Rehabilitation robots are physically assistive devices whose primary function is not communicative, and which are not perceived by patients as social entities. There is a wide range of robots used for physical rehabilitation purposes, such as robots for limb rehabilitation [10], for gait rehabilitation [11], for ankle rehabilitation [12], etc.

However, the new robots can also establish therapeutic human relationships, for example in the context of occupational therapy and the treatment of Parkinson's or Alzheimer's disease. At the simplest level, social robots can ensure basic functional elements of interpersonal communication and socialization. On a more sophisticated level, they can act as observers that accurately detect and prioritise people, objectives, and contextual attributes relevant to reasoned and ethical therapeutic interaction.

A new emerging strand of telerehabilitation is that of wearable technology. Thanks to the miniaturization of electronic components, many types of wearable devices have been developed for applications in health and wellbeing, such as the Apple's Health Kit and Google Fit. The application of wearable technology to monitor older adults and subjects with chronic conditions at home and in community settings is multiplying, while the integration of wearable and ambient sensors has made significant progress. In the logic of the Internet of Things, the new health sensors are smart, since they are able to connect with other devices and share data.

A wide range of telerehabilitation applications is now available for stroke patients, both during and after hospitalization. During hospitalization, the interventions consist of individual counseling sessions and group health education sessions focusing on medication, on healthy behaviour, and on anxiety and depression. Following their discharge from hospitals, patients are checked at home via the internet and are encouraged to perform exercises and physical activity.

Telerehabilitation programmes are demonstrating their effectiveness in specific areas of discouraging unhealthy eating habits, encouraging the cessation of smoking, and treating anxiety and depression for patients following a stroke.

In physical telerehabilitation programs, there is a general consensus as to the usefulness of gaming technology. A game-based approach can effectively motivate patients to follow their course of treatment and 
exercise. Gamified health and fitness applications, nowadays, range from those related to quitting smoking to asthma management [13].

\section{Market Trends}

It is widely shared that the telerehabilitation market is growing, and will noticeably increase in the near future (Figure 1), since consumers are naturally inclined to get or make use of 'newfangled' products if they present valuable advantages, for example in terms of greater availability, convenience, and time-saving.
Currently, the telerehabilitation market encompasses four main sectors:

-Control at distance of patients and medical devices;

-Computer-assisted rehabilitation exercises (physical and cognitive);

-Communication between patients, doctors, and caregivers;

-Distance training, either for patients or medical personnel.

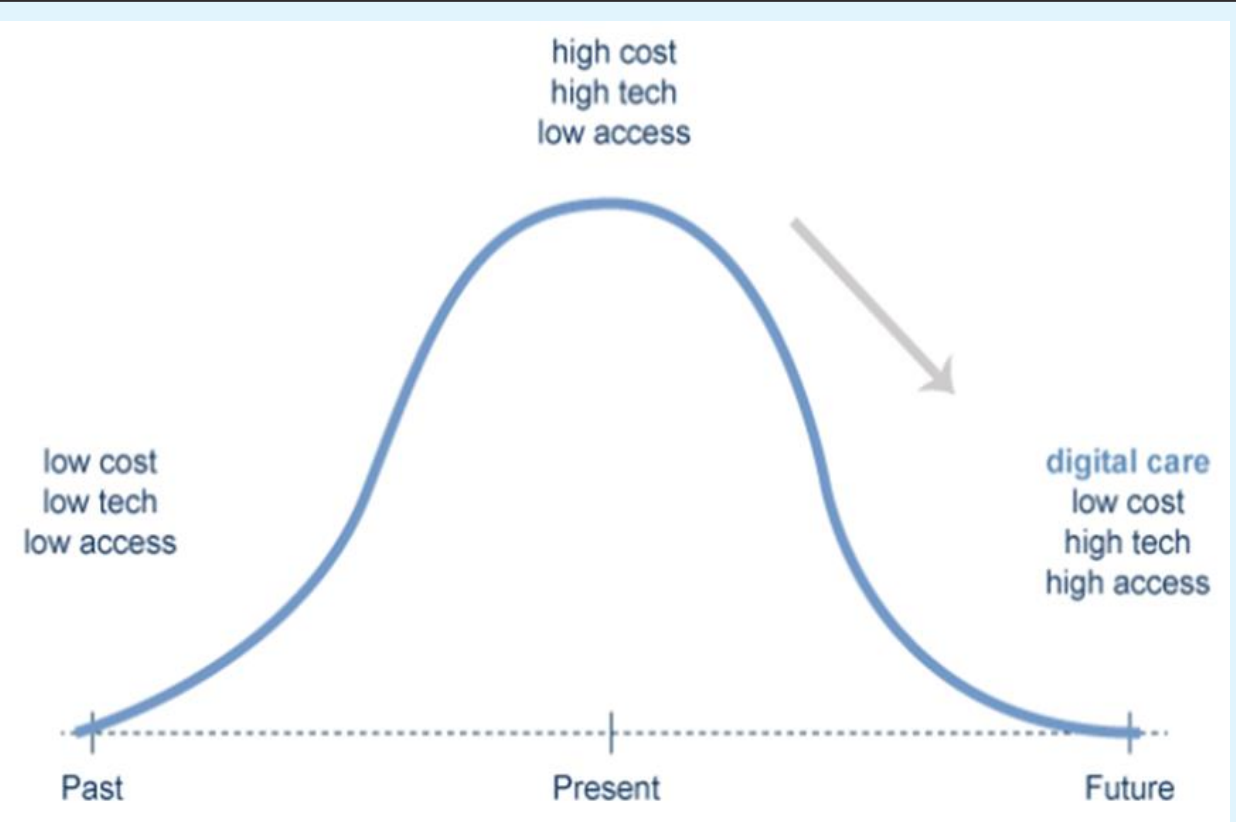

Figure 1: Healthcare innovation market (source: Goldman Sachs Global Investment Research, 2015).

The telerehabilitation market is really vast and includes a large range of products and services, such as:

- Wearable Sensors and Smart Devices for Active and Assisted Living;

- Smart Homes and Living Environments;

- Smartphone Apps, such as Smart Things for Active and Assisted Living;

- Security, Safety, and Privacy - proof Smart Things for Active and Assisted Living;

- Intelligent Data Processing and Predictive Algorithms;

- Cloud Technologies supporting Smart Things for Active and Assisted Living;

- Interoperability and Standardisation Issues;

- Open data for Active and Assisted Living;
- User interfaces for Smart Things for Active and Assisted Living.

A recent report [14] provided a detailed overview of the global telerehabilitation systems market. It reveals that the size of this market can be estimated at $\$ 160$ billion, with a rate growth of approximately $27 \%$ per year.

Some examples of very common products associated with telerehabilitation are:

- Health monitors which continuously monitor pulse, skin temperature, and movement;

- Video monitors;

- Hip protectors; 
- Pressure mats which check if a person mobilises from a bed or a chair;

- Door alerts for patients who wonder at night;

- Movement detectors;

- Balance exercises;
- Teleconferencing.

Data from the QYR Pharma \& Healthcare Research Center (January 2017) confirms the growth trend of the telerehabilitation market in the USA (Figures 2 and 3).

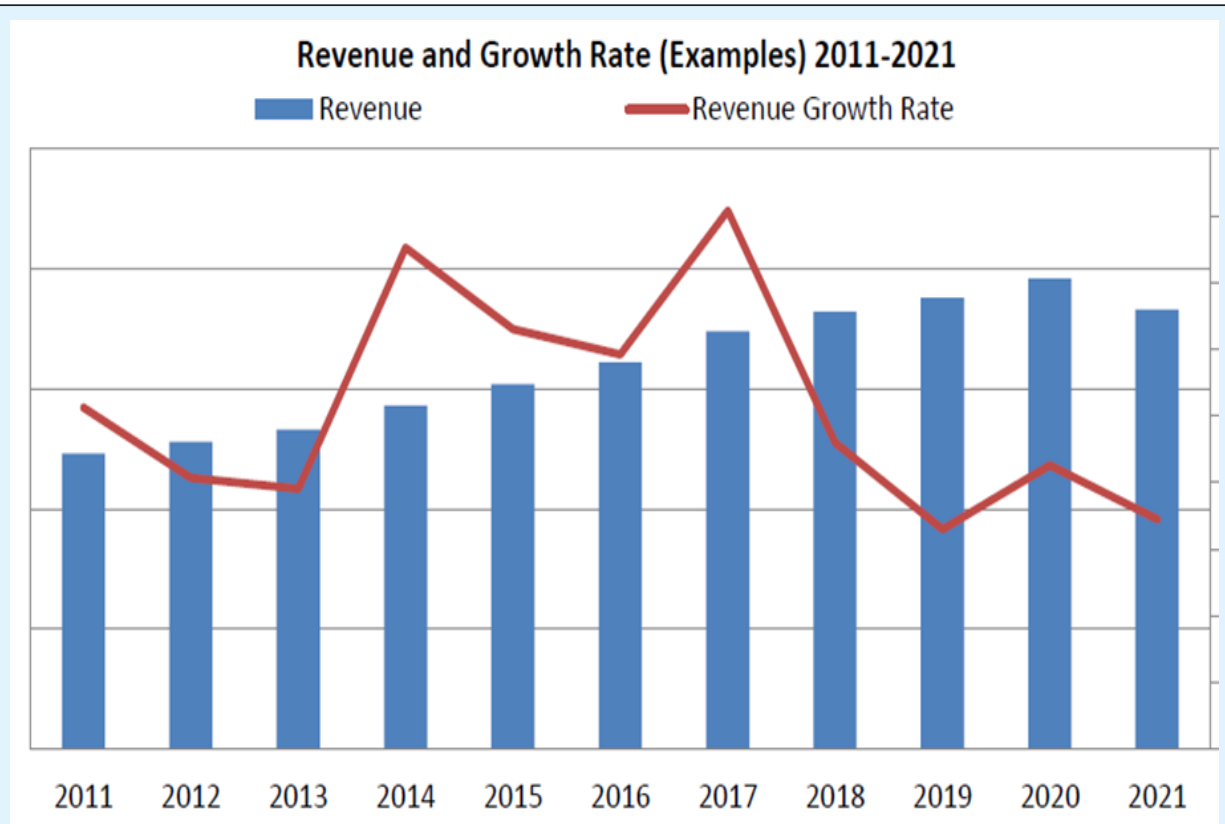

Figure 2: USA telerehabilitation systems revenue and growth rate (source: QYR Pharma \& Healthcare Research Center).

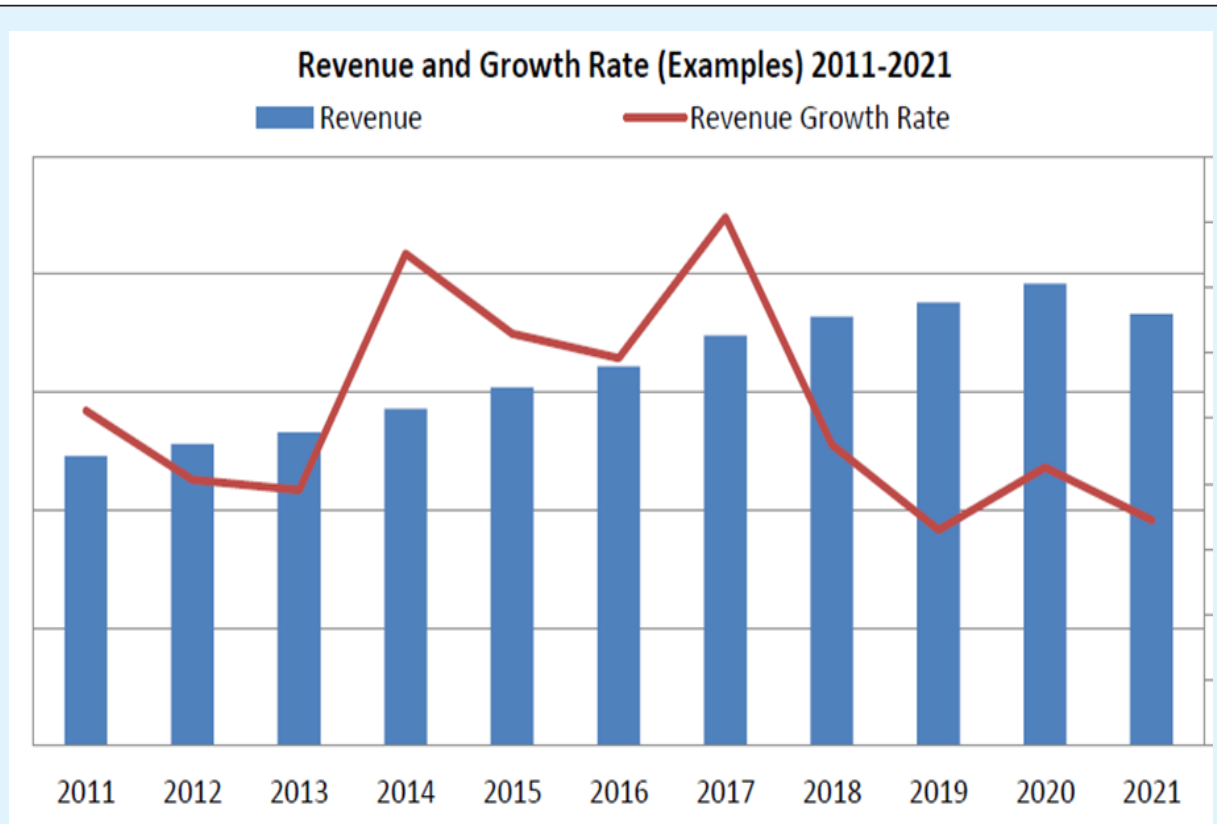

Figure 3: Europe telerehabilitation systems revenue and growth rate. 
Forecasts by Goldman Sachs put the total value of the US telerehabilitation market at $\$ 32.4$ billion, of which $45 \%$ derives from remote patient monitoring; $37 \%$ from telehealth; and $18 \%$ from behavioural modification. Chronic disease management falls entirely within remote patient monitoring. At present, total revenue is estimated in the several hundred millions, with a wide range of company performance, ranging from as low as $\$ 5$ million to just under $\$ 100$ million (Table 1 ).

\begin{tabular}{|c|c|c|c|}
\hline Vertical & Disease State & $\begin{array}{c}\text { Total Savings } \\
\text { Opportunity }\end{array}$ & $\begin{array}{c}\text { Commerical } \\
\text { Opportunity }\end{array}$ \\
\hline $\begin{array}{c}\text { Remote Patient } \\
\text { Monitoring }\end{array}$ & Heart Diseases, COPD/Asthama, Diabetes & $\$ 200+$ billions & $\sim \$ 15$ billion \\
\hline Telehealth & Routine \& Psychological care & $\$ 100+$ billion & $\sim \$ 12$ billion \\
\hline $\begin{array}{c}\text { Behaviour } \\
\text { Modification }\end{array}$ & $\begin{array}{c}\text { Obesity, smoking cessation, overall lifestyle } \\
\text { improvement }\end{array}$ & Indefinitely large & $\sim \$ 6$ billion \\
\hline
\end{tabular}

Table 1: Potential economic impact of ICTs on the healthcare market (source: Goldman Sachs Global Investment Research, 2015).

\section{Telerehabilitation Business}

In one of the first analysis on the economics of telerehabilitation, it was argued that the introduction of telerehabilitation at home was a challenge due to the cost of capital investment [15].

Nowadays, the Internet connectivity and decreasing computer costs make telerehabilitation services more affordable [16] and the research attention focuses on the telerehabilitation business model [17]. Although there is a general agreement that the business model for innovative services/products in healthcare should support the value creation for the whole society (i.e. patients, caregivers, medical personnel and decision makers) rather than exclusively for the firm that provides them [18] the development of an actual business model that ensures effective, sustainable, and value-based telerehabilitation services presents some basic issues. On one hand, there is the problem that telerehabilitation services ought to be harmonized with the current rehabilitation services and with complex and extensive health and social care needs [19]. Telerehabilitation services should be not only userfriendly and cost-effective but also be integrated with existing systems, aligned with existing policies and, in certain cases, act to complement rather than replace faceto-face contact with professionals $[20,21]$. On the other hand, there is the problem of the very rapid evolution of technologies and, as a consequence, the wide diffusion of not integrated self-monitoring technologies that provide readings of step detection, sleep-depth measurements and even heart rate. Most self-monitoring devices are not approved for medical purposes and are not interoperable, but they are low-cost, user-friendly and thus attractive for consumers [22].
In this regard, it is useful to shortly review the company strategies in the field of telerehabilitation.

Companies working in the telerehabilitation sector can be divided into two main categories:

- Big global players that offer a wide range of general purpose solutions, products, and services;

- SMEs that produce specific products and equipment, often included in the offer of the big global players.

There are many companies that produce devices for use in telerehabilitation, either at home or at healthcare structures.

In the last few years, game-based rehabilitation has received wide spread attention, and some commercially available games consoles, such as the Sony Eye Toy and Nintendo Wii, have been widely used to support telerehabilitation experiments [23]. Online games are even now deemed to be a good way of motivating patients, since most of the rehabilitation treatments are based on repetitive tedious exercises [24,25]. Recently, Microsoft has entered into the telerehabilitation sector with their Kinect motion sensor, originally for the Xbox 360, which was developed to create an active and immersive gaming experience. Kinect technology enables users to control and interact with their consoles using gestures and spoken commands, and provides a human skeletal tracking system that can be used for musculoskeletal health exercises. It includes an SDK (software development kit), APIs (Application Program Interface), and tools to develop customised solutions.

Motmi (http://motmi.rehab/en/), an innovative IT and software development company, has developed a virtual rehabilitation platform that enables physical 
rehabilitation in patients via a 3-D gaming environment with real-time feedback, which automatically supervises movement using body tracking technology (for head, trunk, and limbs)provided by the Microsoft Kinect sensor.

Some well-known corporations are starting to invest in the telerehabilitation sector. One such company is Royal Philips, which introduced its Aging Well Services in 2016. This is a collection of solutions aimed at helping elderly people to live healthily and independently in their own homes. Its corporate strategy is based on the creation of partnerships with innovative companies specialized in telerehabilitation. One of its partners is Respond Well, a digital health company that either provides home rehabilitation services or empowers physicians, physical therapists, and healthcare providers to assist their patients remotely. Respond well uses an online gamified approach to rehabilitation, using on-screen digital instructors and incentives.

There are other companies that are specialised in home doctor check-ups, such as Conversahealth.com, or that use cloud platforms to provide patients with direct connections to their health care team, such as Health123.com. Their services have been designed in compliance with the USA Health Insurance Portability and Accountability Act (HIPAA).

Other companies, such as Mindmaze, have developed hospital-based solutions for motor rehabilitation based on computer-based training exercises.

Within the wide context of companies operating at different levels in the telerehabilitation sector, it is possible to identify:

- Companies that provide products for health facilities and/or household purposes;

- Companies that offer services and that may use products developed by different suppliers.

It is notable that many of these products are the result of the assembly of components and solutions applied from various areas, such as those for the analysis of patient movement that combines the data processed from smart sensors and image analysis.

There is another aspect that should not be underestimated: in the medical field, products are functional to treatments or services. This means that the development of telerehabilitation solutions cannot disregard the accurate analysis of organisational problems associated with the delivery of services. In other words, a telerehabilitation service is, in essence, a proceduralised and controlled medical process. Simply put, the remote check-in not only involves features such as the assembling and management of devices that measure and transmit data but must also ensure patient confidentiality and respect medical procedures and responsibilities.

\section{Issues and Challenges}

There is an ever-increasing experimentation in the various branches of telerehabilitation, but the underlying issue is how to transform these experiments into effective services that can both be delivered by healthcare structures, and be reimbursed by health insurance providers.

It is not easy to measure the actual impact of telerehabilitation solutions, since the organization of a service is, clearly, rather different to the experimentation of a solution. Furthermore, the sustainability of a telerehabilitation service depends on many organizational factors, including the training of the medical personnel and the users. Consequently, the online delivery of rehabilitation services may often make it necessary to rethink them, and to define new rehabilitation protocols related to them.

Thus, the questions arise of what the requirements are for including a telerehabilitation treatment in the scope of official healthcare services (for example, in the case of virtual reality home therapy for post-stroke patients), and of how to ensure the quality control of such a treatment. Furthermore, what proof of success must be provided in order to convince insurance companies to cover the cost of such treatments?

These are the fundamental questions that must be asked when considering the merits of a new telerehabilitation service.

A recent study conducted in the USA on the coverage and reimbursement of state telemedicine services reported that only 7 states are known to reimburse for telerehabilitation services in their Medicaid plans, whilst of the 25 states that cover home telemedicine, only 10 (Alaska, Colorado, Delaware, Iowa, Kentucky, Maine, Nebraska, Nevada, New Mexico, and Tennessee) reimburse for telerehabilitation services within the scope of home health benefits [26]. 
In addition, despite the great advances that have been made in telerehabilitation and the very positive figures relating to the size of the market for this sector, there are currently some big challenges and problems that affect the technical and organizational sphere of telerehabilitation.

The interoperability and integration of the various different devices is the principal technical issue, whilst an important organizational issue concerns the balance between the types of treatment that are better delivered at healthcare facilities, compared to those that can be advantageously delivered at a distance $[27,28]$.

The problem of interoperability and integration can be summarized in the question of how we can utilize the data created by smart devices and smart sensors to build effective telerehabilitation services. This is one of the biggest challenges, since we need to bear in mind that in order to use this data we need to be able to store and access it. This is not a simple question, since devices and sensors commonly have independent program interfaces, and use different data formats and diverse semantic rules.

Another challenging question concerns the use of commercial game platforms $[29,30]$. There is a general consensus that, despite their accommodative design aimed at a diverse target audience, commercial game systems present some serious deficiencies for application in this field, due to the omission of medical considerations during their development. Typically, they lack the levels of adaptability and integration necessary to satisfy the heterogeneous nature of treatments.

Finally, we must also tackle the issue of security and patient privacy. It is no easy task to ensure security and privacy when different pieces of equipment lack any type of common controls.

\section{Conclusion}

The reduction of transport costs is only one of the advantages of telerehabilitation. In fact, an online rehabilitation system can provide mechanisms that enhance patient-caregiver and patient-doctor communication, since doctors and caregivers as well as patients can receive real-time feedback concerning the performance of rehabilitation treatments. This allows for a quick and ubiquitous assessment of the patients' progress, and an adjustment of their rehabilitation program when necessary.
However, the question remains as to how the environment the patient is in affects the delivery of services. In other words, to what extent does the home environment influence the telerehabilitation result?

Unfortunately we currently have no significant evidence to be able to address this question.

Perhaps, for the moment, creating decentralized telerehabilitation centres could represent an intermediate solution that could enable the maximization of the benefits of remote treatment in a properly designed and controlled environment.

What we can say, then, is that the development of effective telerehabilitation services is not only a question of technology. Much also depends on the development of appropriate models of corporate structures and investments, and it will also be necessary to define proper criteria for the acceptance of telerehabilitation treatments.

\section{References}

1. World Health Organization (2010) Telemedicine: opportunities and developments in member states. Report on the second global survey on eHealth. World Health Organization.

2. de la Torre-Díez I, López-Coronado M, Vaca C, Aguado JS, de Castro C (2015) Cost-utility and costeffectiveness studies of telemedicine, electronic and mobile health systems in the literature: a systematic review. Telemed J E Health 21(2): 81-85.

3. Behkami NA, Daim TU (2016) Background Literature on the Adoption of Health Information Technologies. In Healthcare Technology Innovation Adoption. Springer International Publishing pp. 9-35.

4. Liu L, Stroulia E, Nikolaidis I, Miguel-Cruz A, Rincon AR (2016) Smart homes and home health monitoring technologies for older adults: A systematic review. Int j Med Inform 91: 44-59.

5. Nelson R, Staggers N (2017) Health informatics: An interprofessional approach. Elsevier Health Sciences.

6. Lai JC, Woo J, Hui E, Chan WM (2004) Telerehabilitation - a new model for communitybased stroke rehabilitation. J Telemed Telecare 10(4): 199-205. 
7. Langberg H, Lindahl MP, Kidholm K, Dinesen B (2014) Telerehabilitering. Ugeskr Læger 176(10): 944-947.

8. Marzano G, Lubkina V, Rizakova L (2015) Delivering social telerehabilitation services. In Society, Integration, Education. Proceedings of the International Scientific Conference 4: 457-467.

9. Marzano G (2017) Social telerehabilitation, Encyclopedia of Information Science and Technology 4th (Edn.) Chapter 516, IGI Global.

10. Maciejasz P, Eschweiler J, Gerlach-Hahn K, JansenTroy A, Leonhardt S (2014) A survey on robotic devices for upper limb rehabilitation. J Neuroeng Rehabil 11: 3.

11. Bortole M, Venkatakrishnan A, Zhu F, Moreno JC, Francisco GE, et al. (2015) The H2 robotic exoskeleton for gait rehabilitation after stroke: early findings from a clinical study. Journal of neuroengineering and rehabilitation 12(1): 54.

12. Jamwal PK, Hussain S, Ghayesh MH, Rogozina SV (2016) Impedance control of an intrinsically compliant parallel ankle rehabilitation robot. IEEE Transactions on Industrial Electronics 63(6): 1-1.

13. Proffitt R (2015) Gamification in Rehabilitation: Finding the "Just-Right-Challenge". Handbook of Research on Holistic Perspectives in Gamification for Clinical Practice, pp: 132-157.

14. Market Research Report (2016) The global virtual rehabilitation and telerehabilitation systems industry, QYResearch.

15. Dhurjaty S (2004) The economics of telerehabilitation. Telemed J E Health 10(2): 196-199.

16. Paone S, Shevchik G (2013) Making a business case for eHealth and teleservices. In Telerehabilitation pp: 297-309.

17. Wade VA, Taylor AD, Kidd MR, Carati C (2016) Transitioning a home telehealth project into a sustainable, large-scale service: a qualitative study. BMC health services research 16: 183.

18. Osterwalder A, Pigneur Y (2010) Business model generation: a handbook for visionaries, game changers, and challengers. John Wiley \& Sons.
19. LeRouge CM, Tulu B, Wood S (2016) Project Initiation for Telemedicine Services. In E-Health and Telemedicine: Concepts, Methodologies, Tools, and Applications, (chapter 1), IGI-Global, USA pp: 1-24.

20. Mistry H, Garnvwa H, Oppong R (2014) Critical appraisal of published systematic reviews assessing the cost-effectiveness of telemedicine studies. Telemed J E Health 20(7): 609-618.

21. De Raeve P, Gomez S, Hughes P, Lyngholm T, Sipilä M, et al. (2016) Enhancing the provision of health and social care in Europe through eHealth. Int Nurs Rev 64(1): 33-41.

22. Leth S, Hansen J, Nielsen OW, Dinesen B (2017) Evaluation of Commercial Self-Monitoring Devices for Clinical Purposes: Results from the Future Patient Trial, Phase I. Sensors (Basel) 17(1): 211.

23. Chang YJ, Han WY, Tsai YC (2013) A Kinect-based upper limb rehabilitation system to assist people with cerebral palsy. Research in developmental disabilities 34(11): 3654-3659.

24. Geurts L, Vanden Abeele V, Husson J, Windey F, Van Overveldt M, et al. (2011) Digital games for physical therapy: fulfilling the need for calibration and adaptation. In Proceedings of the fifth international conference on Tangible, embedded, and embodied interaction, ACM, pp: 117-124.

25. Moraiti A, Vanden Abeele V, Vanroye E, Geurts L (2015) Empowering occupational therapists with a DIY-toolkit for smart soft objects. In Proceedings of the Ninth International Conference on Tangible, Embedded, and Embodied Interaction, ACM, USA, pp: 387-394.

26. Thomas L, Capistrant G (2016) State telemedicine gaps analysis: coverage and reimbursement. American Telemedicine Association.

27. Reeder B, Chung J, Stevens-Lapsley J (2016) Current telerehabilitation research with older adults at home: an integrative review. J Gerontolo Nurs 42(10): 1520.

28. Van Rooij T, Marsh S (2016) eHealth: past and future perspectives. Personalized Medicine 13(1): 57-70.

29. Andrade KDO, Fernandes G, Martins J, Roma VC, Joaquim RC, et al. (2013) Rehabilitation robotics and serious games: An initial architecture for 
simultaneous players. In Biosignals and Biorobotics Conference (BRC), 2013 ISSNIP, IEEE, pp: 1-6.

30. Yates M, Kelemen A, SikLanyi C (2016) Virtual reality gaming in the rehabilitation of the upper extremities post-stroke. Brain inj 30(7): 855-863.
31. QYR Pharma Healthcare Research Center (2017) Global virtual rehabilitation and telerehabilitation systems consumption, QY Research. 